SCIENTIFIC REPORT

\title{
Differential expression of connective tissue growth factor in microglia and pericytes in the human diabetic retina
}

\author{
E J Kuiper, A N Witmer, I Klaassen, N Oliver, R Goldschmeding, R O Schlingemann
}

Br J Ophthalmol 2004;88:1082-1087. doi: 10.1136/bjo.2003.032045

\begin{abstract}
Background/aim: Connective tissue growth factor (CTGF) stimulates extracellular matrix formation, fibrosis, and angiogenesis. It has a role in the pathogenesis of diabetic nephropathy and possibly in diabetic retinopathy (DR): in cultured retinal vascular cells CTGF is induced by VEGF-A. To further characterise this role the authors investigated CTGF expression in normal and diabetic human retina.

Methods: CTGF expression patterns were studied by immunohistochemistry in the retina of eyes of 36 diabetic persons and 18 non-diabetic controls and compared with markers of endothelial cells (CD31, PAL-E), pericytes (NG2), astrocytes (GFAP), and microglia (CD45).

Results: In the retina, distinct and specific staining of CTGF was observed in microglia, situated around or in close vicinity of retinal capillaries. In the control cases, sporadic staining of pericytes was also observed within the vascular wall. In contrast, in the retina of people with diabetes, CTGF staining in microglia was decreased and staining in pericytes was increased. This pattern of predominantly pericyte staining was observed in 20 out of 36 diabetic cases and in one out of 18 controls. The altered CTGF staining patterns in the diabetic cases did not correlate to staining of PAL-E, a marker of retinal vascular leakage associated with DR.

Conclusions: The study shows that CTGF is expressed in microglia in the normal retina whereas in a large subset of diabetic persons, CTGF expression shifts to microvascular pericytes. This altered CTGF expression pattern appears unrelated to manifest DR and may therefore represent a preclinical retinal change caused by diabetes. The results suggest a distinct, but as yet unidentified, role of CTGF in the pathogenesis of diabetic retinopathy.
\end{abstract}

A preclinical hallmark of early diabetic retinopathy (DR) is the thickening of the capillary basement membrane (BM) resulting from increased production or decreased breakdown of collagen IV, laminin, fibronectin, and other compounds. ${ }^{12}$ In galactose fed rats retinal BM thickening can partially be prevented by downregulation of fibronectin synthesis. This is associated with a reduction of pericyte loss and acellular capillaries, suggesting an important role of BM thickening in the pathogenesis of DR. ${ }^{3}$

Connective tissue growth factor (CTGF) is a member of the CCN family of growth factors, which includes CTGF, Cyr 61, Nov, WISP 1, WISP2, and WISP3. ${ }^{4-6}$ CTGF is important in scarring, wound healing, and in a number of pathological conditions associated with fibrosis. ${ }^{7-10}$ It mainly functions in these processes as a potent inducer of extracellular matrix (ECM) by stimulating production of collagen type $1, \alpha 5$ integin, and fibronectin, ${ }^{11}{ }^{12}$ and as a potent angiogenic factor. ${ }^{6}{ }^{13-16}$ CTGF mRNA expression is induced by TGF- $\beta$ in several cell types, but also by advanced glycation end products (AGEs), ${ }^{17}$ by high glucose (in human mesangial cells, a specialised type of pericytes in the glomerulus), ${ }^{18} 19$ and by vascular endothelial growth factor-A (VEGF-A) in retinal endothelial cells and pericytes. ${ }^{20}$

As all these CTGF inducing factors are positively associated with the pathogenesis or progression of diabetic microvascular complications, such as nephropathy and retinopathy, ${ }^{121} 22$ it is possible that CTGF is also involved in these conditions. Indeed, in early diabetic nephropathy, CTGF mRNA is upregulated in mesangial cells and in podocytes in the human glomerulus. ${ }^{43}$

We hypothesise that CTGF plays a role in the early pathogenesis of diabetic retinopathy in its capacity to induce ECM production leading to capillary BM thickening. In order to explore this hypothesis we investigated expression patterns of CTGF protein in the human retina in diabetic people and normal controls.

\section{MATERIALS AND METHODS}

\section{Human eyes}

The eyes of 18 people without and 36 people with DM ( 14 with DM type 1, 22 people with DM type 2; table 1) were obtained from the Corneabank Amsterdam (the Netherlands), after removal of corneal buttons for transplantation.

Intact eyes were snap frozen in isopentane and stored at $-80^{\circ} \mathrm{C}$ until used. The use of human material was in accordance with the Declaration of Helsinki on the use of human material for research.

\section{Immunohistochemistry}

Tissue blocks of the posterior half of the frozen human globes were cut using a standard protocol. Air dried serial cryostat sections ( $10 \mu \mathrm{m}$ thick) of one tissue block containing midperipheral and central retina of one eye of each patient were fixed in cold acetone for 10 minutes, postfixed for 2 minutes in Zamboni's fixative ( $2 \%$ paraformaldehyde in a saturated picric acid solution), and stained by an indirect immunoperoxidase procedure. For this purpose, sections were incubated for 20 minutes in phosphate buffered saline containing $0.1 \%$ sodium azide and $0.3 \% \quad \mathrm{H}_{2} \mathrm{O}_{2}$ to quench endogenous peroxidase activity. In order to reduce non-specific staining, sections were incubated for 15 minutes in phosphate buffered saline containing $10 \%$ normal goat serum and $0.01 \%$ saponin (Sigma, St Louis, MO, USA). Subsequently, serial sections were incubated overnight at $4{ }^{\circ} \mathrm{C}$ with the following monoclonal antibodies: a human monoclonal antiCTGF antibody labelled with digoxigenin (DIG) (1:200) (FibroGen Inc, San Francisco, CA, USA), the antiendothelial

Abbreviations: AGEs, advanced glycation end products; BM, basement membrane; CTGF, connective tissue growth factor; DM, diabetes mellitus; DR, diabetic retinopathy; ECM, extracellular matrix; TGF- $\beta$, transforming growth factor- $\beta$; VEGF-A, vascular endothelial growth factor-A. 


\begin{tabular}{|llllll|}
\hline \multicolumn{4}{l}{ Table 1} & \multicolumn{4}{l|}{ Characteristics of the human eyes } \\
\hline Human eyes ( $\mathbf{n}$ ) & $\begin{array}{l}\text { Age range } \\
\text { (years) }\end{array}$ & $\begin{array}{l}\text { Mean } \\
\text { (years) }\end{array}$ & $\begin{array}{l}\text { Postmortem } \\
\text { time (hours) }\end{array}$ & $\begin{array}{l}\text { Mean } \\
\text { (hours) }\end{array}$ \\
\hline Controls (18) & $46-79$ & 65 & $11-33$ & 20 \\
DM type 1 (14) & $50-88$ & 69 & $5-26$ & 20 \\
DM type 2 (22) & $60-83$ & 72 & $5-32$ & 18 \\
\hline
\end{tabular}

antibodies PAL-E $(1: 1000)^{24} 25$ and EN-4 (against CD31, 1:500; Sanbio, Uden, the Netherlands), ${ }^{26-28}$ to CD64 (against Fc gamma RI, a marker for monocytes, l:100; Dako, Glostrup, Denmark), ${ }^{29}$ to GFAP (to identify astrocytes, 1:100; Sigma, Zwijndrecht, the Netherlands), to CD45 (against LCA, a marker of microglia, 1:200; Dako, Glostrup, Denmark), to HLA-DR (to detect MHC-II, a marker of microglia, 1:4000; Becton Dickinson, San Jose, CA, USA), ${ }^{30}$ to CD68 (human macrophage antigen, 1:1500; Dako, Glostrup, Denmark), ${ }^{30-32}$ and antibody 225.28 S to NG2 (human melanoma proteoglycan or HMW-MAA), a marker of pericytes, 1:300). ${ }^{33}$ As negative controls, primary antibody was omitted. Sections incubated with the DIG-labelled CTGF antibody were subsequently incubated with a monoclonal mouse anti-DIG antibody (1:300) followed by polyHRP goat antimouse immunoglobulins for 30 minutes. All other sections were directly incubated with the poly-HRP goat antimouse immunoglobulins for 30 minutes. Peroxidase activity was visualised using 3-amino-9-ethyl carbazole (AEC, red colour) or 3,3-diaminobenzidine (DAB, brown colour) with $0.01 \% \mathrm{H}_{2} \mathrm{O}_{2}$ as activator. Rinsing the sections with distilled water terminated colour development. Sections were counterstained with haematoxylin.

\section{Data analysis}

For each antibody, two independent observers examined three masked non-serial sections taken from a standardised tissue sample, encompassing the mid-peripheral to the central part of the retina of each patient. The length of each retina section was approximately $5 \mathrm{~mm}$, so in total $15 \mathrm{~mm}$ of retina was examined per subject.

The pattern and distribution of CTGF staining was studied in detail in the retina by light microscopy. To further identify CTGF positive cells, CTGF staining patterns were compared with serial sections stained for pericytes (anti-NG2), endothelial cells (anti-CD31), microglia (anti-CD45 and anti-HLA-DR), and astrocytes (anti-GFAP). A macrophage marker (anti-CD68) was used to distinguish between quiescent and activated microglia.

The various CTGF staining patterns were compared with staining for PAL-E, a marker of capillary leakage associated with diabetic retinopathy. ${ }^{25}{ }^{28}$ Retinal microvascular staining for PAL-E was graded semiquantitatively as follows: 0, no staining; 1, sporadic staining (less than three positive microvessels per $5 \mathrm{~mm}$ section); 2, patchy staining (localised areas with staining microvessels, but less than $50 \%$ of vessels being positive); or 3, uniform staining (more than 50\% of vessels being positive).

In addition, based on initial examinations, CTGF staining was semiquantified in the capillaries in and adjacent to the inner nuclear layer. For this purpose, at the $40 \times$ magnification, the number of CTGF positive cells with a definite localisation in the neuroretinal parenchyma adjacent to blood vessels of the inner nuclear layer, but outside the vascular basal lamina, and the number of CTGF positive cells definitely localised within the vascular basal lamina were counted in the three non-serial sections (fig 1).

All data were analysed using the statistical program SPSS 7.0 (SPSS Inc, Chicago, IL, USA). The non-parametric Mann-

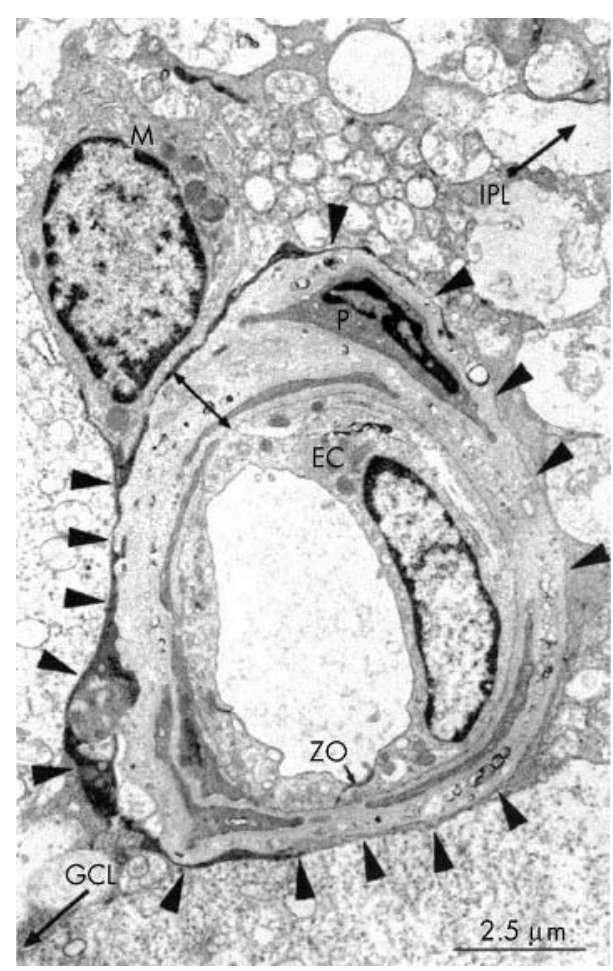

Figure 1 Electron micrograph showing the cell types of the vascular complex in adult human retina. Arrowheads indicate the perivascular glia limitans (basal lamina) of a retinal vessel. A paravascular microglia cell $(M)$ is situated adjacent to the glia limitans (to the left) and contributes processes to it. Endothelial cells (EC) and a single pericyte (P) are present. A zonulae occludens junction (ZO) is present between endothelial cells. Arrows indicate the orientation of the ganglion (GCL) cell and inner plexiform layers (IPL). Reprinted from J M Provis. Development of the primate retinal vasculature. Prog Retin Eye Res 2001;20:799-821, with permission from Elsevier Science.

Whitney test was used to calculate the mean differences in number of pericytes or number of microglia between diabetics and controls. Mean differences between the PALE score and the number of pericytes (and microglia) were calculated with the non-parametric Kruskal-Wallis test. A p value of $<0.05$ was considered significant. Mean difference in the number of cases with a pericyte pattern or microglia pattern between diabetics and controls was calculated by " $\chi$ " crosstabs".

\section{RESULTS}

In the human retina, the CTGF positive cells were mostly located in the vicinity of or directly associated with blood vessels, which were clearly outlined in serial sections stained with anti-CD31. We distinguished two different patterns of CTGF staining in the inner nuclear layer-outside and inside the vascular basement membrane-which was recognised in the sections by its differential refractive properties. The CTGF positive cells located outside the vascular basement membrane often had a stellate or dendritic morphology, suggesting that these cells represented microglia (fig 2A, C, and E). Comparison with the staining pattern for CD45, a microglia marker (fig 2G), in serial sections, identified these CTGF positive cells as microglia. The cellular staining pattern of CD68, a marker of activated microglia and macrophages, also resembled CTGF staining, indicating that activated microglia and possibly also macrophages expressed CTGF (fig 2I). The astrocyte marker GFAP stained Müller cells and perivascular astrocytes in a pattern quite different from the CTGF staining distribution (fig 3). 
The distribution and staining characteristics of the CTGF positive cells within the vascular basal lamina (fig 2B, D, and F) were compared with markers for endothelial cells and pericytes in serial sections. In most instances, these CTGF positive cells were located within the vascular wall in a
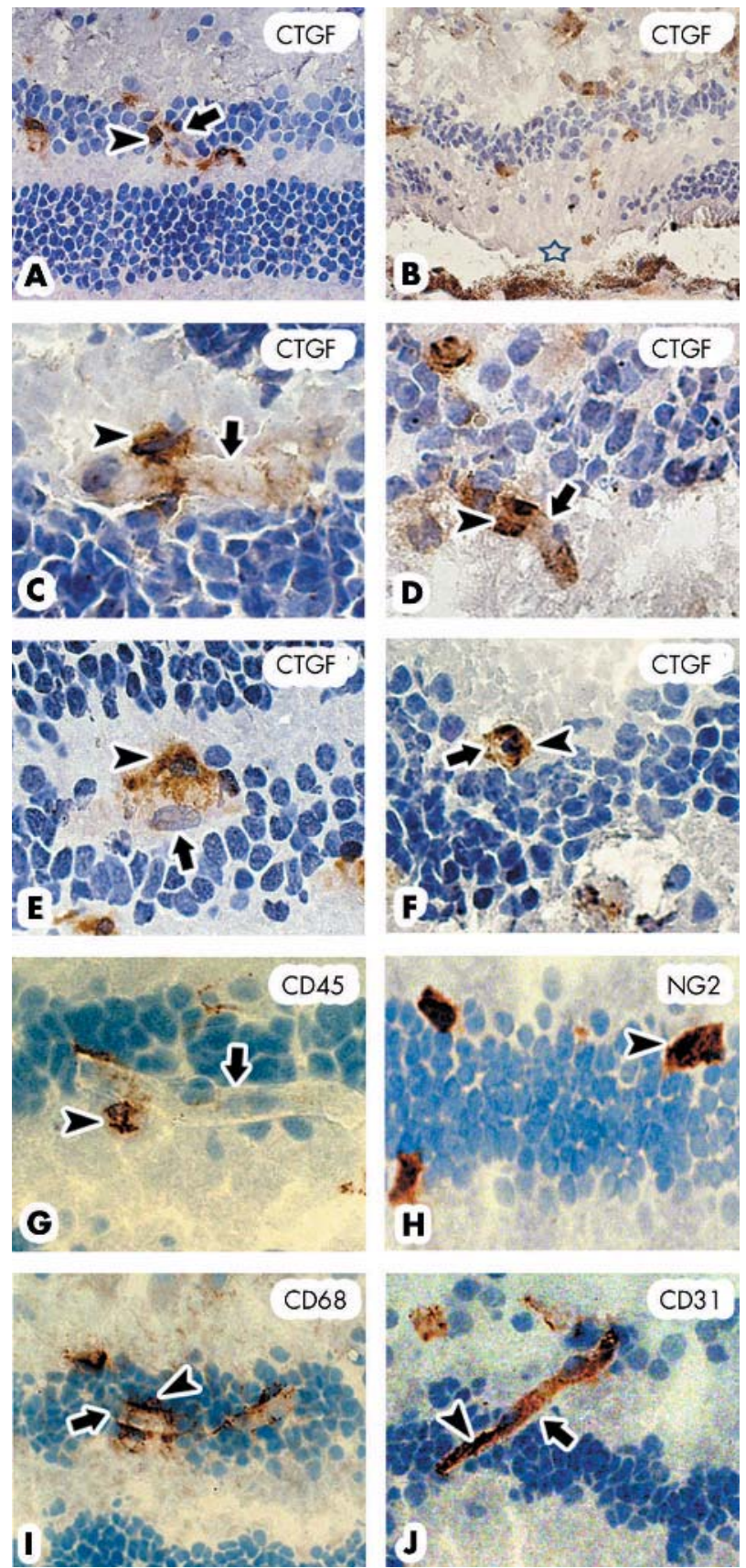

Figure 2 Cryosections showing the different CTGF staining patterns in normal and diabetic human retina. Note the difference in localisation of the CTGF positive cells (arrowheads) outside the vascular basement membrane (indicated by arrows) in the normal human retina ( $A, C$, and $E$ ) and inside the vascular basement membrane (indicated by arrows) of the diabetic human retina (B, D, and F). Staining of microglia markers CD45 (G) and CD68 (I) in normal human retina identifies the CTGF positive cells outside the vascular basal lamina (arrowheads in $A, C$, and $E$ ) as microglia (arrowheads in $G$ and I). Staining of pericyte marker NG2 $(H)$ in capillary fragments in diabetic retina identifies the CTGF positive cells inside the vascular basement membrane (arrowheads in $B$ $D$, and $F$ ) as pericytes (arrowheads in $H$ ). B and D show CTGF staining of pericytes in a retina that has received laser treatment (laser scar indicated by star).

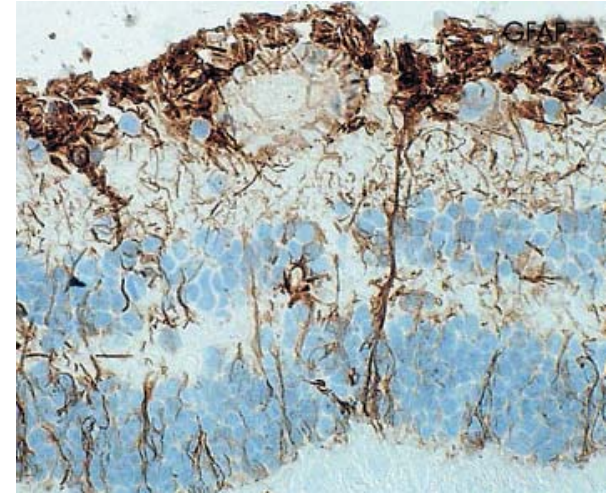

Figure 3 Cryosection of human diabetic retina stained for GFAP, showing staining of astrocytes and Müller cells. Note the difference in staining pattern compared to CTGF staining in figure 2.

similar pattern as pericytes identified by staining of NG2 (fig $2 \mathrm{H}$ ), although it was evident that many pericytes did not stain for CTGF. Occasional CTGF immunoreactivity in endothelial cells (fig $2 \mathrm{~J}$ ) could not be excluded because of the limited resolution of light microscopy.

Quantitative analysis of the total number of CTGF positive cells that could be clearly localised within the vascular basal lamina (pericytes) or in the vicinity of the vascular wall, but outside the basal lamina (microglia), revealed a strikingly different pattern in the retina of diabetic people when compared with controls. Although there was no significant difference according to the total CTGF positive cells counted between diabetic subjects (mean (SD 6.8) 14.6) and controls (mean (SD 6.0) 13.6), the number of CTGF positive cells identified as pericytes was significantly higher in the diabetic group (median 7.0; range 0-23) compared with controls (median 1.5; range $1-6)(\mathrm{p}<0.001)$ (fig 4A). Furthermore the number of CTGF positive cells identified as microglia was significantly lower in the diabetic group (median 4.5; range 0-28) compared with controls (median 11.6; range 2-23) $(\mathrm{p}<0.01)$ (fig 4B).

In fact, two basic patterns of CTGF staining were revealed when the number of CTGF stained pericytes were expressed as a percentage of the total number of CTGF positive cellsthat is, a predominant microglial pattern of CTGF positive cells ( $<40 \%$ of the total CTGF+ cells in a pericyte localisation) and a predominant pericyte staining pattern $(>60 \%$ of the total number of CTGF positive cells in a pericyte localisation). A predominant pericyte staining pattern for CTGF strongly correlated with the presence of diabetes: of the 21 retinas with a pattern of predominantly pericyte staining, 20 were from diabetic subjects $(\mathrm{p}<0.001)$ (eight cases with DM type 1 and 12 cases with DM type 2). A predominant microglia pattern was seen in 16 out of 18 controls and in 14 out of 36 diabetic retinas (five cases with DM type 1 and nine cases with DM type 2). A mixed pattern of CTGF positive pericytes and CTGF positive microglia was observed in two diabetic retinas and in one control case. All five diabetic patients who were known to have had laser therapy showed a predominant pericyte pattern of CTGF staining.

To investigate the possible relation between staining of microglia and pericytes and the presence or absence of diabetic retinopathy, serial sections were stained for the antiendothelial antibody PAL-E. PAL-E is a marker associated with vascular leakage and VEGF receptor-2 expression in diabetic retinopathy. ${ }^{25}{ }^{28}$ In concordance with previous observations, a pattern of patchy or uniform staining of PAL-E in retinal vessels strongly correlated with the presence of diabetes $(p<0.0001)$. Notably, PAL-E staining of the 

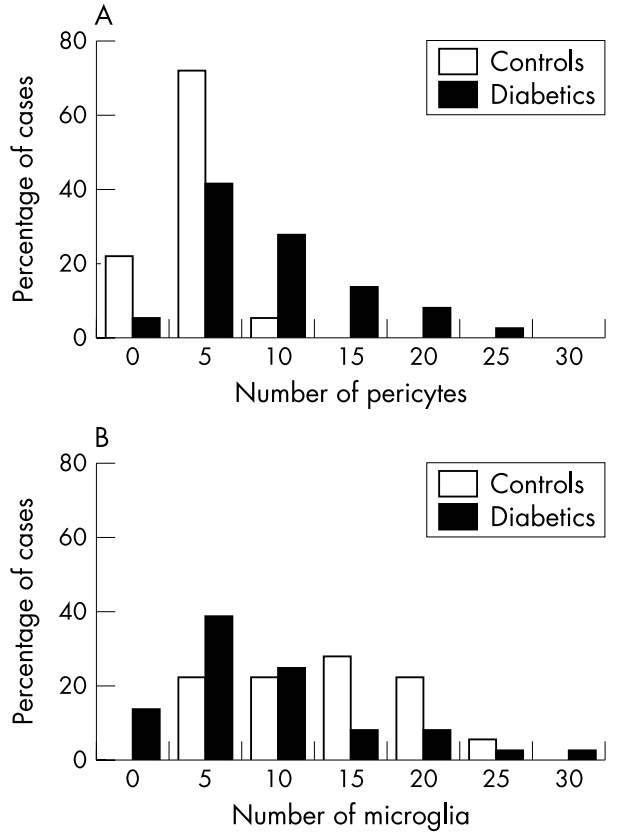

Figure 4 Distribution of pericyte (A) and microglia (B) counts in the inner nuclear layer of retina of control (open bars) and diabetic (solid bars) subjects. Note the higher number of CTGF positive pericytes $(p<0.001)$ and lower number of CTGF positive microglia $(p<0.001)$ in diabetic subjects compared with controls.

diabetic cases did not correlate with either the predominant pericyte or with the predominant microglial distribution pattern of CTGF staining, suggesting that increased CTGF expression in pericytes in DM is not related to the occurrence of retinal vascular leakage or the presence of diabetic retinopathy.

\section{DISCUSSION}

We have shown retinal CTGF expression at the protein level, employing a highly specific monoclonal antibody that does not cross react with other proteins, including other CCN family members (unpublished results). Immunohistochemical staining with this antibody yielded a distinct and specific cellular cytoplasmic staining of CTGF in the retina, consistent with local cellular expression of this protein. The observations of our descriptive study show that in the normal human retina, CTGF is expressed in paravascular microglia. However, in the retina of diabetic subjects, expression in microglia is significantly decreased whereas expression of CTGF in microvascular pericytes is significantly increased. In our series of cases, this yielded two main patterns of CTGF expression, either predominant staining of microglia or predominant staining of pericytes. The predominant pericyte staining pattern was almost exclusively correlated with the presence of diabetes (a schematic representation of our findings is depicted in fig 5).

We investigated CTGF staining in a series of postmortem tissues derived from globes donated anonymously for cornea transplantation. The type of diabetes of the patients from whom we derived these globes is known, but no ophthalmological information on presence or absence of eye diseases such as diabetic retinopathy is available. Histopathological evidence of preretinal neovascularisation as a sign of proliferative DR was not observed in any of these cases. In five cases a history of laser treatment was noted, but whether this was focal or panretinal treatment remains unknown. Each of these five cases had a predominant pericyte pattern of CTGF staining.

We further investigated whether altered CTGF expression in diabetes was associated with $\mathrm{DR}$ by examining the correlation of CTGF expression in pericytes or microglia with the extent of vascular PAL-E staining, a marker associated with local retinal vascular leakage. ${ }^{28}$

Although PAL-E staining cannot be regarded as a direct marker for DR, we have previously shown that PAL-E staining, which is normally absent from blood-retinal barrier endothelium, is distributed in a patchy or uniform distribution in the vasculature of a large subset of diabetic retinas. It correlates spatially with markers of vascular leakage ${ }^{28}$ and expression of VEGFR-2, ${ }^{25}$ the main receptor of VEGF-A, an important mediator of vascular leakage in clinical DR. ${ }^{35} \mathrm{PAL}-$ E can therefore be regarded as a marker of retinal vascular changes associated with clinical DR.

Interestingly, despite the apparent association with a history of laser treatment, we found no correlation of any parameter of changed CTGF expression with PAL-E staining, suggesting that altered CTGF expression in microglia and pericytes in diabetes is unrelated to microvascular leakage and established clinical DR. In fact, altered CTGF expression seems to be evenly distributed in our diabetic cases irrespective of PAL-E staining. Therefore, these changes may be related to the preclinical early changes in the retina that eventually lead to clinical DR.

CTGF has been shown to be upregulated in the retina of diabetic rats, a model of preclinical DR. ${ }^{36}$ In preclinical DR, basement membrane thickening and pericyte death are histopathological hallmarks. ${ }^{1}$ Together with endothelial cell death these are believed to be the result of metabolic changes caused by hyperglycemia and advanced glycation end products (AGEs). ${ }^{137}$ In vitro, CTGF mRNA is induced by AGEs $^{17}$ and high glucose. ${ }^{18}{ }^{19}$ AGEs induce fibronectin, a component of the retinal $\mathrm{BM}^{38}$ in dermal fibroblasts via upregulation of CTGF. ${ }^{39}$ Thus, CTGF may well be involved in $\mathrm{BM}$ thickening in preclinical DR, and this notion is supported
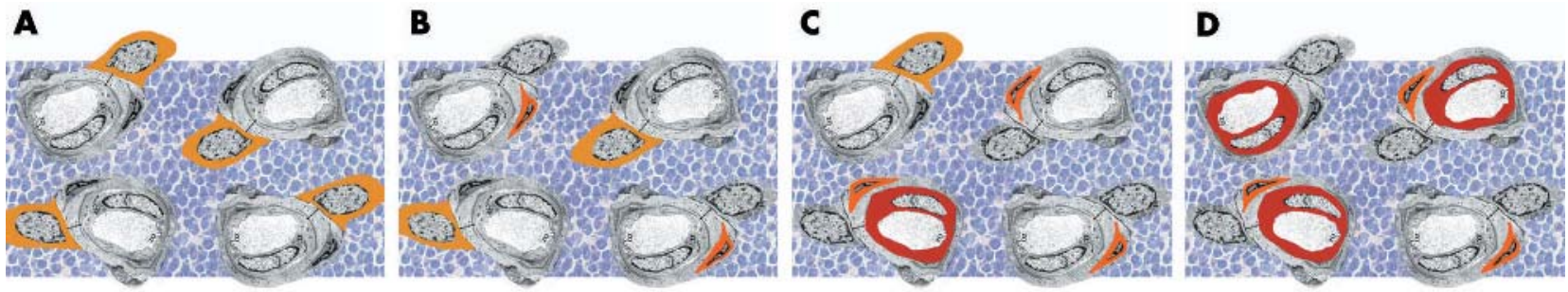

Figure 5 Tentative model of the CTGF staining patterns during the development of diabetic retinopathy, based on the results of this study. Progressive degrees of non-proliferative DR are indicated by an increase in PAL-E positive endothelial cells (red). (A) Control subject with no PAL-E staining, showing only CTGF+ microglia (yellow). (B, C, and D) Diabetic subjects with or without PAL-E staining, showing a decrease of CTGF+ microglia (yellow) and increase of CTGF+ pericytes (orange). 
by our finding that CTGF is overexpressed in pericytes in the human diabetic retina, irrespective of changes related to clinical DR like vascular leakage.

CTGF overexpression in pericytes in the diabetic retina is in line with the reported increase of CTGF expression in mesangial cells of the renal glomerulus (a type of pericyte) in diabetic nephropathy in vivo, ${ }^{40}{ }^{41}$ and with the induction of CTGF by glucose and AGEs observed in mesangial cells in vitro. ${ }^{19}{ }^{42}$ Overexpression of CTGF in cultured human aortic smooth muscle cells, a cell type closely related to pericytes and mesangial cells, induces apoptosis by activating caspase $3{ }^{43} 44$ Therefore, CTGF may also be involved in pericyte apoptosis which is a characteristic of early DR.

Our study shows for the first time that CTGF is expressed in the human retina. In previous reports, CTGF expression (induced by VEGF) was shown in cultured bovine retinal endothelial cells and pericytes. ${ }^{20}$ In our study, no evident endothelial expression was observed which may be due to the considerable phenotypic and functional differences between retinal endothelial cells in vivo and in vitro. ${ }^{45}$ The constitutive expression of CTGF in paravascular microglia in the normal retina suggests an as yet undefined role in retinal microvascular physiology. In the light of the known functions of CTGF in other cells and tissues, it is tempting to speculate that microglia derived CTGF may be involved in retinal matrix or vascular basement membrane homeostasis in normal conditions.

Although our results, by their descriptive nature, do not allow a precise understanding of the function of CTGF in the retina, they do suggest a possible role of CTGF both in retinal physiology and in the pathogenesis of DR.

\section{ACKNOWLEDGEMENTS}

This study was supported by a grant from the Diabetes Fonds Nederland (grant 2001.042) and Edward and Marianne Blaauwfonds. The authors kindly thank Claudia Trischberger for expert technical assistance.

\section{Authors' affiliations}

E J Kuiper, A N Witmer, I Klaassen, R O Schlingemann, Ocular Angiogenesis Group, Department of Ophthalmology, Academic Medical Center, University of Amsterdam, Amsterdam, the Netherlands R Goldschmeding, Department of Pathology, Academic Medical Center of Utrecht, Utrecht, the Netherlands

N Oliver, FibroGen Inc, San Francisco, CA, USA

Commercial relations: E J Kuiper, none; A N Witmer, none; I Klaassen, none; $\mathrm{N}$ Oliver, Fibrogen $\mathrm{F}$; $\mathrm{R} O$ Schlingemann, Fibrogen $\mathrm{F} ; \mathrm{R}$ Goldschmeding, Fibrogen F.

Correspondence to: Dr R O Schlingemann, Department of Ophthalmology, Academic Medical Center, Meibergdreef 15, $1105 \mathrm{AZ}$ Amsterdam, the Netherlands; r.schlingemann@amc.uva.nl

Accepted 1 January 2004

\section{REFERENCES}

1 Lorenzi M, Gerhardinger C. Early cellular and molecular changes induced by diabetes in the retina. Diabetologia 2001:44:791-804.

2 Spirin KS, Saghizadeh M, Lewin SL, et al. Basement membrane and growth factor gene expression in normal and diabetic human retinas. Curr Eye Res 1999; 18:490-9.

3 Roy S, Sato T, Paryani G, et al. Downregulation of fibronectin overexpression reduces basement membrane thickening and vascular lesions in retinas of galactose-fed rats. Diabetes 2003;52:1229-34.

4 Goldschmeding R, Aten J, Ito Y, et al. Connective tissue growth factor: just another factor in renal fibrosis? Nephrol Dial Transplant 2000;15:296-9.

5 Grotendorst GR, Lau LF, Perbal B. CCN proteins are distinct from, and should not be considered members of, the insulin-like growth factor-binding protein superfamily. J Clin Endocrinol Metab 2001;86:944-5.

6 Lau LF, Lam SC. The CCN family of angiogenic regulators: the integrin connection. Exp Cell Res 1999;248:44-57.

7 Grotendorst GR. Connective tissue growth factor: a mediator of TGF-beta action on fibroblasts. Cytokine Growth Factor Rev 1997;8:171-9.
8 Moussad EE, Brigstock DR. Connective tissue growth factor: what's in a name? Mol Genet Metab 2000;71:276-92.

9 Igarashi A, Nashiro K, Kikuchi K, et al. Significant correlation between connective tissue growth factor gene expression and skin sclerosis in tissue sections from patients with systemic sclerosis. J Invest Dermatol 1995; 105:280-4.

10 Igarashi A, Nashiro K, Kikuchi K, et al. Connective tissue growth factor gene expression in tissue sections from localized scleroderma, keloid, and other fibrotic skin disorders. J Invest Dermatol 1996:106:729-33.

11 Frazier K, Williams S, Kothapalli D, et al. Stimulation of fibroblast cell growth, matrix production, and granulation tissue formation by connective tissue growth factor. J Invest Dermatol 1996;107:404-11.

12 Duncan MR, Frazier KS, Abramson S, et al. Connective tissue growth factor mediates transforming growth factor beta-induced collagen synthesis: downregulation by CAMP. FASEB J 1999;13:1774-86.

13 Shimo T, Nakanishi T, Nishida T, et al. Involvement of CTGF, a hypertrophic chondrocyte-specific gene product, in tumor angiogenesis. Oncology $2001 ; 61: 315-22$

14 Shimo T, Kubota S, Kondo S, et al. Connective tissue growth factor as a major angiogenic agent that is induced by hypoxia in a human breast cancer cell line Cancer Lett 2001;174:57-64.

15 Babic AM, Chen CC, Lau LF. Fisp12/mouse connective tissue growth factor mediates endothelial cell adhesion and migration through integrin alphavbeta3, promotes endothelial cell survival, and induces angiogenesis in vivo. Mol Cell Biol 1999:19:2958-66.

16 Shimo T, Nakanishi T, Nishida T, et al. Connective tissue growth factor induces the proliferation, migration, and tube formation of vascular endothelial cells in vitro, and angiogenesis in vivo. J Biochem (Tokyo) 1999: 126:137-45.

17 Twigg SM, Chen MM, Joly AH, et al. Advanced glycosylation end products up-regulate connective tissue growth factor (insulin-like growth factor-binding protein-related protein 2) in human fibroblasts: a potential mechanism for expansion of extracellular matrix in diabetes mellitus. Endocrinology 2001:142:1760-9.

18 Murphy M, Godson C, Cannon S, et al. Suppression subtractive hybridization identifies high glucose levels as a stimulus for expression of connective tissue growth factor and other genes in human mesangial cells. J Biol Chem 1999:274:5830-4.

19 Paradis V, Perlemuter G, Bonvoust F, et al. High glucose and hyperinsulinemia stimulate connective tissue growth factor expression: a potential mechanism involved in progression to fibrosis in nonalcoholic steatohepatitis. Hepatology $2001 ; 34: 738-44$.

20 Suzuma K, Naruse K, Suzuma I, et al. Vascular endothelial growth factor induces expression of connective tissue growth factor via KDR, Fltl, and phosphatidylinositol 3-kinase- akt-dependent pathways in retinal vascular cells. J Biol Chem 2000;275:40725-31.

21 Roy S, Cagliero E, Lorenzi M. Fibronectin overexpression in retina microvessels of patients with diabetes. Invest Ophthalmol Vis Sci 1996;37:258-66

22 Roy S, Maiello M, Lorenzi M. Increased expression of basement membrane collagen in human diabetic retinopathy. J Clin Invest 1994;93:438-42.

23 Ito $Y$, Aten J, Bende RJ, et al. Expression of connective tissue growth factor in human renal fibrosis. Kidney Int 1998;53:853-61

24 Schlingemann RO. Monoclonal antibody PAL-E specific for endothelium. Lab Invest 1985;52:71-6.

25 Witmer AN, Blaauwgeers HG, Weich HA, et al. Altered expression patterns of VEGF receptors in human diabetic retina and in experimental VEGFinduced retinopathy in monkey. Invest Ophthalmol Vis Sci 2002;43:849-57.

26 Provis JM, Sandercoe T, Hendrickson AE. Astrocytes and blood vessels define the foveal rim during primate retinal development. Invest Ophthalmol Vis Sci 2000;41:2827-36.

27 Schlingemann RO. Differential expression of markers for endothelial cells, pericytes and basal lamima in the microvasculature of tumors and granulation tissue. Am J Pathol 1991;138:1335-47.

28 Schlingemann RO, Hofman P, Vrensen GF, et al. Increased expression of endothelial antigen PAL-E in human diabetic retinopathy correlates with microvascular leakage. Diabetologia 1999;42:596-602.

29 Allen E, Bakke AC, Purtzer MZ, et al. Neutrophil CD64 expression: distinguishing acute inflammatory autoimmune disease from systemic infections. Ann Rheum Dis 2002;61:522-5.

30 Provis JM, Penfold PL, Edwards AJ, et al. Human retinal microglia: expression of immune markers and relationship to the glia limitans. Glia $1995 ; 14: 243-56$

31 Provis JM, Diaz CM, Penfold PL. Microglia in human retina: a heterogeneous population with distinct ontogenies. Perspect Dev Neurobiol 1996;3:213-22.

32 Yuan L, Neufeld AH. Activated microglia in the human glaucomatous optic nerve head. J Neurosci Res 2001;64:523-32.

33 Ozerdem U, Monosov E, Stallcup WB. NG2 proteoglycan expression by pericytes in pathological microvasculature. Microvasc Res 2002;63:129-34.

34 Schlingemann RO. Expression of the high molecular weight melanomaassociated antigen by pericytes during angiogenesis in tumors and in healing wounds. Am J Pathol 1990:136:1393-405.

35 Schlingemann RO, van Hinsbergh VW. Role of vascular permeability factor/ vascular endothelial growth factor in eye disease. Br J Ophthalmol 1997;81:501-12

36 Tikellis C, Cooper ME, Twigg SM, et al. Connective tissue growth factor is upregulated in the diabetic retina: amelioration by ACE inhibition. Endocrinology 2003.

37 Garner A. Histopathology of diabetic retinopathy in man. Eye 1993;7:250-3. 
38 Roy S, Zhang K, Roth T, et al. Reduction of fibronectin expression by intravitreal administration of antisense oligonucleotides. Nat Biotechnol 1999; 17:476-9.

39 Twigg SM, Joly $\mathrm{AH}$, Chen MM, et al. Connective tissue growth factor/IGFbinding protein-related protein-2 is a mediator in the induction of fibronectin by advanced glycosylation end-products in human dermal fibroblasts. Endocrinology 2002; 143:1260-9.

40 Wang S, Denichilo M, Brubaker C, et al. Connective tissue growth factor in tubulointerstitial injury of diabetic nephropathy. Kidney Int 2001;60:96-105.

41 Riser BL, Cortes P. Connective tissue growth factor and its regulation: a new element in diabetic glomerulosclerosis. Ren Fail 2001;23:459-70.
42 Blom IE, van Diik $A J$, Wieten $L$, et al. In vitro evidence for differentia involvement of CTGF, TGF $\beta$, and PDGF-BB in mesangial response to injury. Nephrol Dial Transplant 2001;16:1139-48.

43 Hishikawa K, Nakaki T, Fujii T. Connective tissue growth factor induces apoptosis via caspase 3 in cultured human aortic smooth muscle cells. Eur J Pharmacol 2000;392:19-22.

44 Hishikawa K, Oemar BS, Tanner FC, et al. Connective tissue growth factor induces apoptosis in human breast cancer cell line MCF-7. J Biol Chem 1999;274:37461-6.

45 Bachetti T, Morbidelli L. Endothelial cells in culture: a model for studying vascular functions. Pharmacol Res 2000;42:9-19. 